\title{
Interface behavior between carbon nanotubes and asphalt
}

\author{
LI Dong Sheng ${ }^{1,2, a}$ \\ 1Shanxi Road and Bridge Construct Co., LTD, Taiyuan Shanxi, 030006, PR China \\ Shanxi Sanwei Huabang Group Co., LTD, Hongtong Shanxi, 041603, PR China \\ aemail: 1398482772@qq.com and1064145779@qq.com
}

\begin{abstract}
Keywords: Interface mechanical behavior; Carbon nanotubes; Asphalt; First-principle; Molecular dynamic simulation

Abstract. The objective aims to study the bonding, deformation and failure behavior of carbon nanotubes-asphalt interface using molecular simulations. The electronic structure and electrical properties of carbon nanotubes modified asphalt (CNMA) were investigated by first-principle molecular dynamic including density of state and band structure. Tensile simulation through uniform stress were measured and the stress responses were obtained to analyze the interface mechanical behavior. It indicated that carbon nanotubes (CNTs) could improve remarkably the electrical and mechanical property of asphalt. Molecular dynamic simulation results showed that failure modes of the interface between CNTs and asphalt turned out to be mainly adhesive failure rather than cohesive failure. The interface failure strength and maximum deformation were affected by carbon nanotubes and temperature that was consistent with the viscoelastic behavior of asphalt binder.
\end{abstract}

\section{Introduction}

Asphalt is widely applied in road and bridge deck pavement due to the good performance of pavement in asphalt mixtures[1-2]. Asphalt is the byproduct of petroleum refinement and a complex collides that about 90-95wt percent of asphalt is comprised of carbon and hydrogen and it is also called hydrocarbon [3-4]. There are many weakness of asphalt such as fatigue cracking, rutting and oxidation aging[5-7]. one of the main solution is adding modifications. Owing to the size and interface effect, nano modification have been successful applied in asphalt and the rutting resistance and thermal cracking resistance of modified asphalt is better than virgin asphalt[8-10]. Nano carbon nanotubes (CNTs) is one of the unique modification. CNTs modified asphalt could improve the aging resistance and thermal cracking resistance and be a kind of potential material of road pavement. However, nano carbon nanotubes modified asphalt (CNMA) exists the interface adhesive weaken.

All manuscripts must be in English, also the table and figure texts, otherwise we cannot publish your paper.

Molecular simulation concluded first principle molecular dynamic and molecular dynamic simulations is a computer simulation of the electronic structure and molecular movement of particles based on the physical principles of atoms and molecules. The trajectories of atoms or molecules are determined by newton's law. There are many researches that have studied the asphalt material using first principle molecular dynamics and molecular dynamic simulation. The population numbers of epoxy modified asphalt were calculated by first principle and molecular dynamic[11]. The solubility of layered double hydroxides modified asphalt were studied by molecular dynamic simulations[12-13]. The three and four components asphalt model were recently established using molecular dynamic simulations and the components schematic structure were drew by Chew-draw software[14]. Physicochemical properties of virgin asphalt were investigated by molecular dynamic simulations[15]. Xu et al[16] indicated the cohesion and adhesion properties of asphalt concrete using molecular dynamics simulation. Actually, investigations on the molecular level can increase the fundamental understanding of the properties of asphalt, as well as the interface mechanical mechanism of CNMA. Moreover, there are few literatures that molecular simulation were used to study the interface mechanical behaviors such as bonding, deformation and failure between CNTs and asphalt. 
In order to better understand the interface mechanical behavior between CNTs and asphalt, molecular simulations were used to calculate the density of state, band structure, interface failure strength and stress responses with experimental verification.

\section{Simulation details and experiments}

\section{Asphalt modeling}

Asphalt is a complex chemical mixture and are three main constituents, i.e., asphaltenes, saturates and resins. According to the literatures, the saturates, aromatics and asphaltenes in asphalt were modeled as straight chain alkane $\mathrm{C}_{22} \mathrm{H}_{46}, 1,7$-dimethyl-naphthalene, Groenzin and Mullin's model $\mathrm{C}_{72} \mathrm{H}_{98} \mathrm{~S}$, respectively. The structural formula of asphaltenes, saturates and resins are shown in Fig.1.

(a)

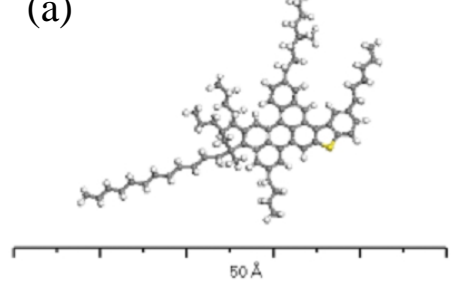

(b)

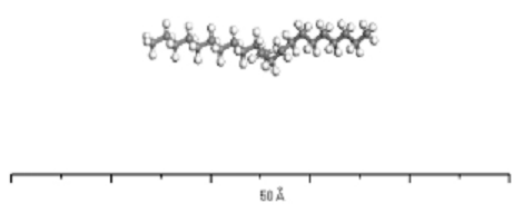

(c)

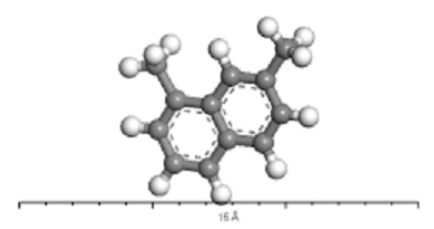

Figure 1 Structural formula of (a) asphaltenes, (b) saturates and (c) resins (carbon atoms are shown Interface modeling in grey, sulfur atoms in yellow, and hydrogen atoms in white)

The asphalt model proposed by Zhou[17] was adopted in this research. carbon nanotubes was come from cleaving graphite using build modules. The interface models of carbon nanotubes-asphalt were built by Layer modular of Materials Studio software package and the interface thickness was $10 \AA$. The schematic structure showed in Fig.2.

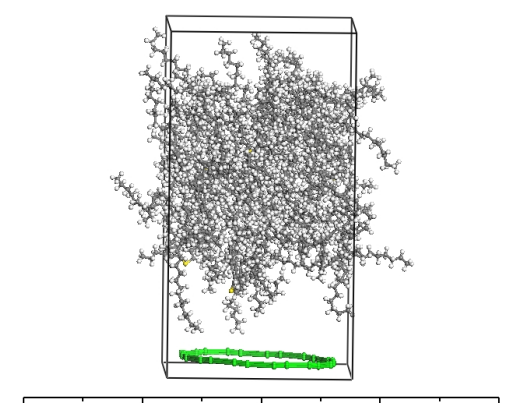

Figure 2 The schematic structure of the interface of carbon nanotubes and asphalt

\section{First-principle calculations}

With the Materials Studio software, first principle were used to calculate the population numbers with CASTEP modular. The generalized gradient approximation (GGA) of density function theory (DFT) was used with Perdew-Burke-Ernzerh (PBE) function and fine convergence precision.

\section{Molecular dynamic simulations}

The force field used in all dynamic simulations and minimization process was the Condensed-phase Optimized Molecular Potentials for Atomistic Simulation Studies (COMPASS) force-field. The initial density of interface was $1.0 \mathrm{~g} / \mathrm{cm}^{3}$. Pressure for all simulations was set at $0 \mathrm{kPa}, 101.325 \mathrm{kPa}(1.0$ atm), 202.650kPa (2.0 atm), $405.300 \mathrm{kPa}(4.0 \mathrm{~atm})$ and $810.600 \mathrm{kPa}(8.0 \mathrm{~atm})$. This study first relaxed to induce the system energy using geometry optimization. Then, relaxed to state equilibrium using NPT ensemble with 1.0fs time step and 100ps simulation steps with different pressure. The stress data were collected each $1 \mathrm{fs}$ and showed certain variations that is probably due the thermal fluctuations in the system. If the simulation period in the molecules moving average were selected too large, some details would be lost, such as the peak stress value and the corresponding separation distance. So, a relative small window was used for moving average. 


\section{Low-temperature scanning}

To understand the interfacial interactions between CNTs and asphalt, the complex modulus were measured by dynamic shear rheology at a fixed frequency of $10 \mathrm{rad} / \mathrm{s}$ and a variable strain, by using the $25 \mathrm{~mm}$ parallel plates with a $1 \mathrm{~mm}$ gap (h) during the temperature range between $-5^{\circ} \mathrm{C}$ and $30^{\circ} \mathrm{C}$.

\section{Results and discussions}

\section{Electronic structure and electrical properties}

The exponential forms of the density of states (DOS) in the interface of CNTs and asphalt were used to predict the conduction properties. In Fig.3, the DOS of CNMA showed that the electron concentrated on $-20 \mathrm{ev}-0 \mathrm{ev}$ and the distribution of DOS was uniform and there appeared width peaks at $-8 \mathrm{ev}-0 \mathrm{ev}$. DOS of CNTs indicated that the $\mathrm{C}$ atoms formed the valence bond and the covalency was strongly. As we well known, the more flat energy band is, the more sharper the DOS peaks are. DOS peaks of asphalt were sharp which revealed that the delocalization were strongly. Compare the DOS of CNTs with the DOS of CNMA, the DOS from -5ev to 10ev changed greatly, which showed that the bond of low energy were forming and the interaction of bond was weaken. Moreover, this simulation result is in accordance with experiments.

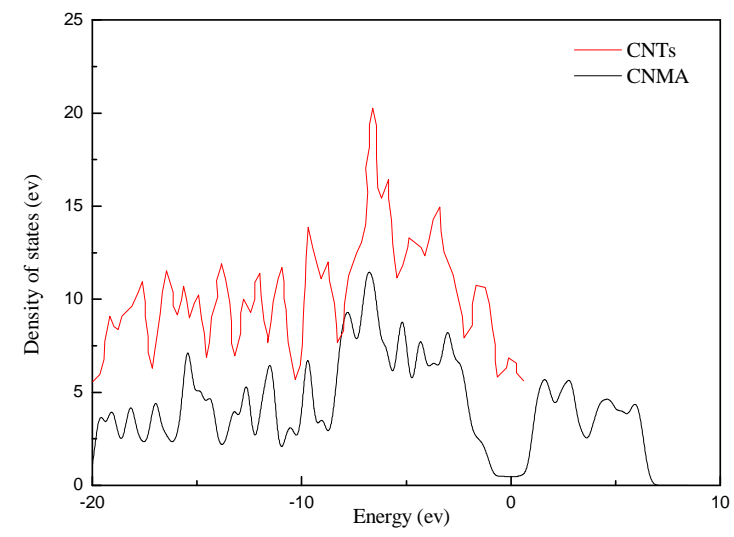

Figure 3 Density of states carbon nanotubes modified asphalt (CNMA) and carbon nanotubes (CNTs)

The band gaps of CNMA and CNTs by using PBE exchange with GGA as the correlation part together with the experimental results are shown in Table 1. As can be seen, in the frame work of pseudo-petential approach, the band gaps results calculated by PBE correlation was bigger results than that of the experimental results.

Table 1 Band gaps of carbon nanotubes and carbon nanotubes modified asphalt

\begin{tabular}{|l|l|l|}
\hline Type & $\begin{array}{l}\text { Band gaps } \\
\text { (simulated results) }\end{array}$ & $\begin{array}{l}\text { Band gaps } \\
\text { (experimental results) }\end{array}$ \\
\hline CNTs & $2.1 \mathrm{ev}$ & $1.8 \mathrm{ev}$ \\
\hline CNsMA & $2.6 \mathrm{ev}$ & $2.5 \mathrm{ev}$ \\
\hline
\end{tabular}

In Fig.4, the calculated results of band structure for CNTs and CNMA are shown. As mentioned before, the energy band structure of CNTs arranged densely and the forbidden band width was 2.1ev; the width between Fermi level and conduction band was 1.2ev, which demonstrated that asphalt did not belong to conductor and was non-conductive material; the forbidden band width of the interface between CNTs and asphalt was 2.3ev, which indicated that CNMA existed conductivity. These effects are more significant for energies between about $-4 \mathrm{ev}$ and $2 \mathrm{ev}$, where the zero energy refers to Fermi level (EF). The experimental values of crossover points at room temperature are about $2.5 \mathrm{ev}$. Our results are in excellent agreement with the experiments. However, using the PBE functional lead to crossover points equal to $2.6 \mathrm{ev}$.

As atoms concentration. Affects the density of states significantly and increases the DOS in the vicinity of the Fermi level and below it down to about 4ev, while density of states of the CNTs at 
conduction band remains almost unchanged, especially for conduction band minima in the $\mathrm{L}$ and $\mathrm{H}$ valley. In contrast, as it can be seen from Fig.4, compared with as atoms in asphalt, $\mathrm{C}$ atoms in asphalt have less effect on the density of states of valence band in the vicinity of the Fermi level, and have further effect on the conduction band. However, the effects of CNTs concentration on valence and conduction band in both cases are mainly due to the $\mathrm{p}$ orbitals, and s orbitals only contribute to conduction band.
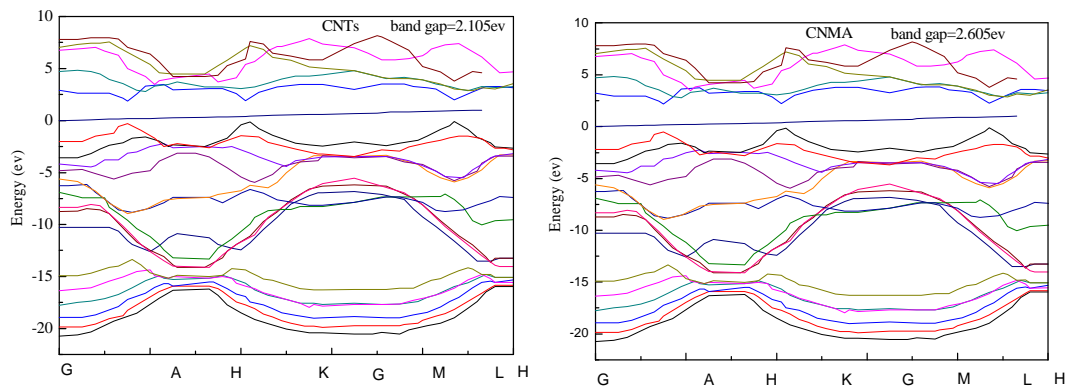

Figure 4 band structure of carbon nanotubes and carbon nanotubes modified asphalt

\section{Interface mechanical behavior Stress-separation responses}

Fig.5 showed the CNMA results of stress-separation relationship during the tension and interface failure process. The applied pressure were $0 \mathrm{~atm}$ and the temperature were controlled at $273 \mathrm{k}\left(0^{\circ} \mathrm{C}\right)$. The stress-displacement relationship can be described that the stress experienced an initial linear increase, which continued up to a peak value at about $2-3 \AA$ displacement. The stress decreased sharply because a reduction of interaction between CNTs and asphalt took place when the impressed pressure was added. The interface stress decreased to zero or a small value in the end, which indicated a final separation between CNTs and asphalt. The stress-separation relationship showed that the adhesive strength of the CNTs and asphalt interface was weaken.

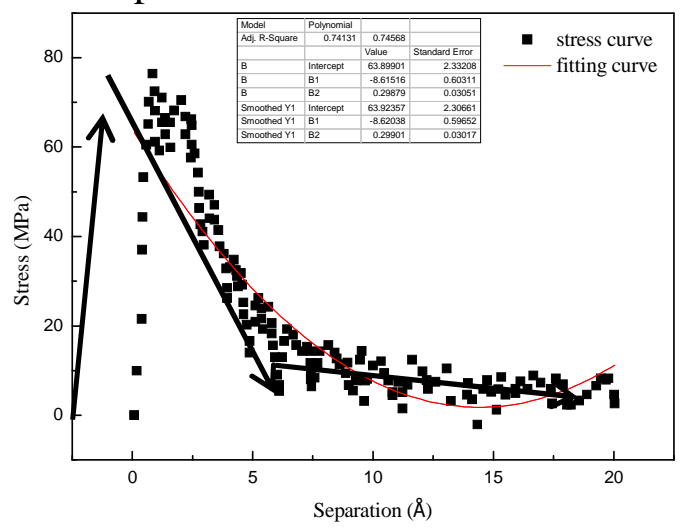

Figure 5 Stress-displacement relationship in tensile simulation

\section{Interface strength and maximum deformation}

Fig.6 showed the fitted tensile strength-displacement curve with an exponential model. The interface strength is defined by the peak value of the fitted tensile strength-displacement curve. The interface strength of $3.8 \mathrm{wt} \%, 7.3 \mathrm{wt} \%, 10.6 \mathrm{wt} \%$ and $13.6 \mathrm{wt} \% \mathrm{CNMA}$ were $78.95 \mathrm{MPa}, 70.85 \mathrm{MPa}, 67.75 \mathrm{MPa}$ and $53.38 \mathrm{MPa}$, respectively at $1 \mathrm{~atm}$. It was noted that compared to the interfacial tensile strength measured from experimental results, the prediction from molecular dynamic simulation was greater than the experimental results. The maximum deformation of $3.8 \mathrm{wt} \%, 7.3 \mathrm{wt} \%, 10.6 \mathrm{wt} \%$ and $13.6 \mathrm{wt} \%$ CNMA were $3.2 \AA, 1.8 \AA, 3.7 \AA$ and $3.5 \AA$, respectively. It revealed that the maximum deformation without any rules and the prediction values were smaller by about eight magnitude. This could be caused by the applied loading pressure used in molecular dynamic simulation that was much smaller due to the constraint of computation time and volume. 


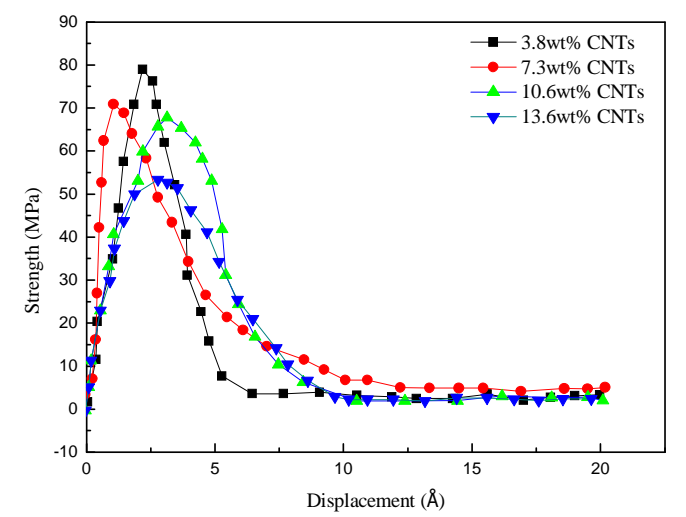

Figure 6 The fitted tensile strength-displacement curve with an exponential model

As shown in Fig.7, the interface strength of CNMA at 0atm, 1atm, 2atm, 4atm and 8atm are $92 \mathrm{MPa}, 86 \mathrm{MPa}, 56 \mathrm{MPa}, 45 \mathrm{MPa}$ and $33 \mathrm{MPa}$, respectively. It indicated that the interface strength of CNMA decreased with the increased loading pressure and the increased speed was remarkably. The

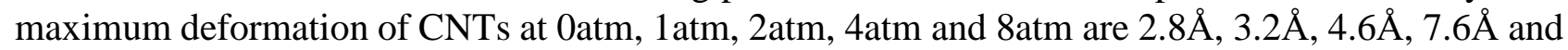
$12.1 \AA$, respectively, which demonstrated that the maximum deformation increased as the loading pressure increased because of the applied loading pressure leading to the volume of CNMA in the interface model compressed. The interface of CNTs and asphalt was unstable and adhesive strength was low at ordinary pressure.

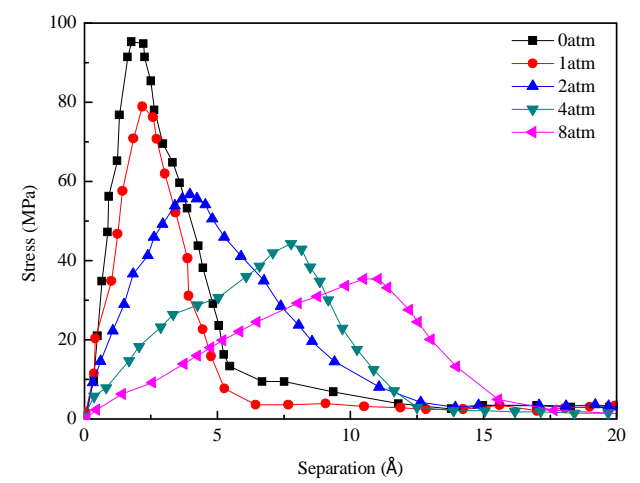

Figure 7 The interface strength of carbon nanotubes modified asphalt

\section{Loading pressure effect}

Five loading pressure were performed to see whether it has effect on the interface behavior. Generally speaking, loading pressure of $0 \mathrm{~atm}, 1 \mathrm{~atm}, 2 \mathrm{~atm}, 4 \mathrm{~atm}$ and $8 \mathrm{~atm}$ were equivalent to stress of $0 \mathrm{~N}$, $9.8751 \times 10^{-11} \mathrm{~N}, 1.9750 \times 10^{-10} \mathrm{~N}, 3.9500 \times 10^{-10} \mathrm{~N}$ and $7.9000 \times 10^{-10} \mathrm{~N}$, respectively, with respect to the original length of asphalt model configuration.

The effects of stress on the stress-strain curves are shown in Fig.8. The different loading pressure can affect the shape of the interface and stress-strain curve, together with the peak value of interfacial tensile strength. It indicated that the interface deformed under smaller pressure or stress levels had a longer separation process before complete interface failure; while the shape of stress-strain curves under the little strain levels are narrower and sharper. Therefore, the loading pressure has more significant effect on the post-peak failure process (plastic stage) as compared to the pre-peak process (linear elastic stage). The separation can be reached when the interfacial stress between CNTs and asphalt reached peak value for the higher stress level. This can be treated as pure adhesive failure for the lower stress level, the separation was defined when the stress was reduced to a small value with some fluctuations. In general, the less the pressure level was applied, the greater the peak stress value was obtained. 


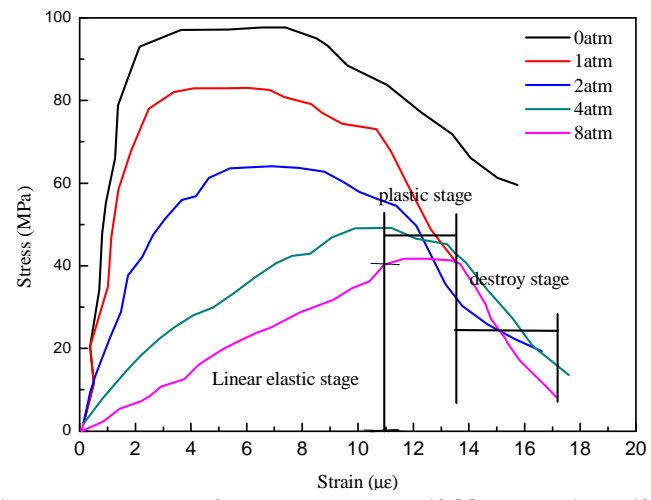

Figure 8 The stress-strain curves at different loading pressure

\section{Interface failure mechanisms}

Interface energy calculation

The interface adhesive energy (Einterface) was used to characterize the adhesive properties of the carbon nanotubes-asphalt interface. In order to better understand the adhesive properties of CNMA at different pressure, the Einterface of CNMA is calculated by the following formula:

$$
E_{\text {interface }}=\left(E_{\text {asphalt }}+E_{\text {surface }}-E_{\text {total }}\right)
$$

where, $E_{\text {aspahlt }}$ is the total energy of asphalt, Esurface is the surface energy of CNTs, Etotal is the total energy of carbon nanotubes modified asphalt.

The interface adhesive properties could be characterized by the absolute value of interface adhesive energy. As shown in Table 2, the interface adhesive energy of 3.8wt\%, $7.3 \mathrm{wt} \%, 10.6 \mathrm{wt} \%$ and $13.6 \mathrm{wt} \%$ CNMA were $-1284.65 \mathrm{kcal} / \mathrm{mol},-1685.21 \mathrm{kcal} / \mathrm{mol}, \quad-2045.43 \mathrm{kcal} / \mathrm{mol}$ and $-2486.98 \mathrm{kcal} / \mathrm{mol}$, respectively. It showed that the adhesive property of carbon nanotubes-asphalt interface increased with the increased CNTs contents.

Table 2. the interface adhesive energy of carbon nanotubes modified asphalt $(\mathrm{kcal} / \mathrm{mol})$

\begin{tabular}{lllll}
\hline Type & Etotal & Esurface & Easphalt & Einterface \\
\hline 3.8wt\%CNMA & 10896.48 & 3939.24 & 5672.59 & -1284.65 \\
7.3wt\%CNMA & 12186.40 & 4714.94 & 5786.25 & -1685.21 \\
10.6wt\%CNMA & 18378.51 & 10463.65 & 5869.43 & -2045.43 \\
13.6wt\%CNMA & 19197.21 & 11173.13 & 5978.65 & -2486.98 \\
\hline
\end{tabular}

\section{Interface interaction}

Complex modulus of CNMA could characterized the interface interaction between carbon nanotubes and asphalt. The more the complex modulus of CNMA is, the higher the interface interaction is. As shown in Fig.9, the complex modulus of CNMA increased with the increased carbon nanotubes content and temperature within limit. It indicated that the interface interaction of CNMA decreased with the increased the carbon nanotubes and temperature within limit. This results were verified with the simulation results. 


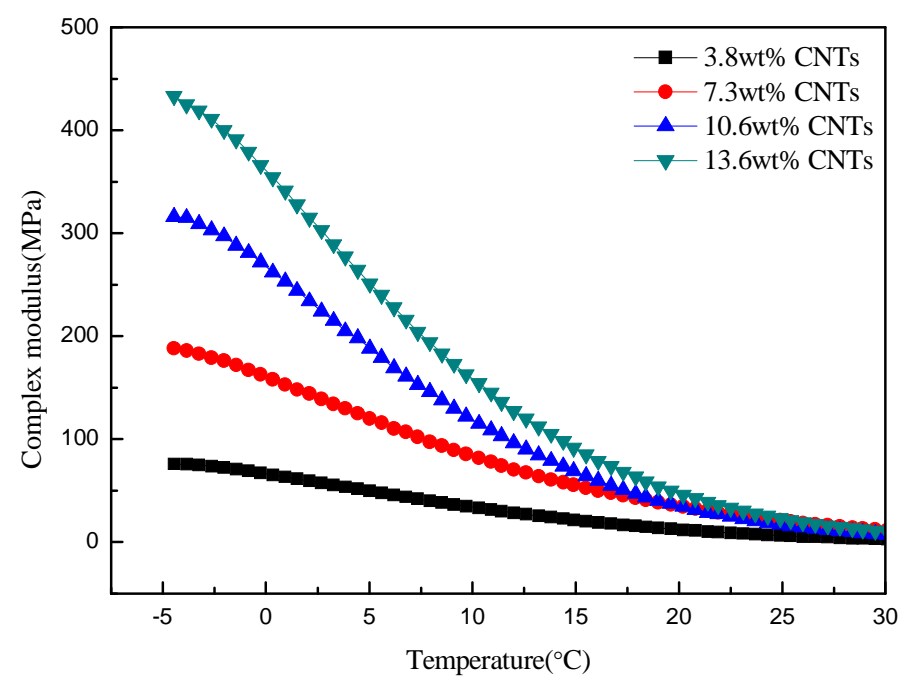

Figure 9 Scanning curves at low temperatures: $-5^{\circ} \mathrm{C}-30^{\circ} \mathrm{C}$

\section{Interface failure modes}

The interface failure mechanisms of $3.8 \mathrm{wt} \%, 7.3 \mathrm{wt} \%, 10.6 \mathrm{wt} \%$ and $13.6 \mathrm{wt} \%$ CNMA were different. In order to better understand the failure mechanism of CNMA, the failure energy $(\Delta \mathrm{E})$ is defined as following formula:

$$
\Delta E=E_{\text {interface }}-E_{\text {cohesive }}
$$

where, $\mathrm{E}_{\text {interface }}$ is the interface adhesive energy of CNMA, Ecohesive is the cohesive energy of asphalt binder. $\Delta$ positive value of $\Delta \mathrm{E}$ represents the failure mechanism is cohesive failure and negative value represents the failure mechanism is adhesive failure.

The cohesive energy of asphalt binder was $14299.40 \mathrm{kcal} / \mathrm{mol}$ in this study. The $\Delta \mathrm{E}$ of $3.8 \mathrm{wt} \%, 7.3 \mathrm{wt} \%, 10.6 \mathrm{wt} \%$ and $13.6 \mathrm{wt} \% \mathrm{CNMA}$ were $-15584.05 \mathrm{kcal} / \mathrm{mol},-15984.61 \mathrm{kcal} / \mathrm{mol}$, $-16344.83 \mathrm{kcal} / \mathrm{mol}$ and $-16786.38 \mathrm{kcal} / \mathrm{mol}$, respectively. It indicated that the failure mechanism of CNMA was adhesive failure, the failure of CNMA decreased with the increased carbon nanotubes addition.

It could be found that the failure modes of the interface between CNTs and asphalt under this condition turned out to be mainly adhesive failure rather than cohesive failure. The asphalt system was stretched at first and micro-voids were initiated in regions of the asphalt network with lower connectivity afterwards, when CNTs displaced apart from the asphalt layer. These micro-voids grew and coalesced with further separation of the CNTs layer.

\section{Conclusion}

We concluded the interface behavior of CNMA and it indicated that failure modes of the interface between CNTs and asphalt turned out to be mainly adhesive failure rather than cohesive failure. The adhesive property of carbon nanotubes-asphalt interface decreased with the increased CNTs contents. Furthermore, the elastic of asphalt changed with the increased contents of CNTs. The experiment results showed that the maximum deformation force increased with the increased content of CNTs. And the change law of tension strength was not irregular. Furthermore, the interface interaction of CNMA decreased with the increased the carbon nanotubes and temperature within limit. All the same time, the simulation and experiments are consistent.

\section{Acknowledgements}

This work was supported by the National Natural Science Foundation of China (No.5180082096) and Science and Technology Department of Shanxi Province International Cooperation 
(No.201603D421027) and the Shanxi Provincial Key Research and Development Program (No.201603D321118).

\section{References}

[1]Mino, G.D. Airey, G. Paola, M.D. et al. (2016) Linear and nonlinear fractional hereditary constitutive laws of asphalt mixtures. Journal of Civil Engineering \& Management, (in press 2015), pp882-889.

[2]White, G. (2016) Shear stresses in an asphalt surface under various aircraft braking conditions. International Journal of Pavement Research \& Technology, 2016, 9, pp89-101.

[3]Yao, H. Dai, Q. and You, Z. (2015) Chemo-physical analysis and molecular dynamics (MD) simulation of moisture susceptibility of nano hydrated lime modified asphalt mixtures. Construction \& Building Materials, 101, pp536-547.

[4]Xu, G. and Wang, H. (2016) Molecular dynamics study of interfacial mechanical behavior between asphalt binder and mineral aggregate. Construction \& Building Materials, 121, pp246-254.

[5]Moreno, F. and Rubio, M.C. (2013) Effect of aggregate nature on the fatigue-cracking behavior of asphalt mixes. Materials \& Design, 47, pp61-67.

[6]Saboo, N. Kumar, P. (2016) Analysis of different test methods for quantifying rutting susceptibility of asphalt binders. Journal of Materials in Civil Engineering, 28, pp112-117.

[7]Mcgovern, M.E. Buttlar, W.G. and Reis, H. (2016) Field assessment of oxidative aging in asphalt concrete pavements with unknown acoustic properties. Construction \& Building Materials, 116, pp159-168.

[8]You, Z. Mills-Beale, J. Foley, J.M. et al. (2011) Nanoclay-modified asphalt materials: preparation and characterization. Construction \& Building Materials, 25, pp1072-1078.

[9]De Melo, J.V.S. and Trichês, G. (2016) Evaluation of rheological behavior and performance to permanent deformation of nanomodified asphalt mixtures with carbon nanotubes. Canadian Journal of Civil Engineering, 35, pp256-262.

[10]Zhang, H.L., Su, M.M. Zhao, S.F. et al. (2016) High and low temperature properties of nano-particles/ polymer modified asphalt. Construction \& Building Materials, 114, pp323-332.

[11]Zhou, X.X. Wu, S.P. Liu, G. et al. (2016) Molecular simulations and experimental evaluation on the curing of epoxy bitumen. Materials \& Structures. 49, pp241-247.

[12]Zhou, X.X. Wu, S.P. Liu, Q. et al. (2015) Effect of surface active agents on the rheological properties and solubility of layered double hydroxides-modified asphalt. Materials Research Innovations, 19, pp978-982.

[13]Takanohashi, T. Iino, M. Nakamura, K. (2002) Evaluation of association of solvent-soluble molecules of bituminous coal by computer simulation. Energy \& Fuels, 8, pp291-297.

[14]Xu, M. Yi, J. Feng, D. et al. (2016) Analysis of adhesive characteristics of asphalt based on atomic force microscopy and molecular dynamics simulation. ACS Applied Materials \& Interfaces, 15 , pp42-48.

[15]Hui, Y. Dai, Q. and You, Z. (2016) Molecular dynamics simulation of physicochemical properties of the asphalt model. Fuel, 164, pp83-93.

[16]Xu, G.J. and Wang, H. (2016) Study of cohesion and adhesion properties of asphalt concrete with molecular dynamics simulation, Computational Materials Science. 112, pp161-169.

[17]Zhou, X.X. Zhang, G.F. Liu, R.M. et al. (2014) Molecular simulations of anti-aging mechanisms on nano-LDHs modified asphalt. Key Engineering Materials, 599, pp198-202. 